\title{
Intra-arterial Drug Delivery to the Ischemic Brain in Rat Middle Cerebral Artery Occlusion Model
}

Fereshteh Azedi' ${ }^{1}$, Masoud Mehrpour², Somaieh Kazemnejad³ ${ }^{3}$ Kazem Mousavizadeh", Amir Hassan Zarnani ${ }^{5,6}$, Mohammad Taghi Joghataei ${ }^{1,7, *}$

${ }^{1}$ Department of Neuroscience Faculty of Advanced Technologies in Medicine, Iran University of Medical Sciences, Tehran, Iran; ${ }^{2}$ Department of Neurology, Faculty of Medicine, Iran University of Medical Sciences, Tehran, Iran; ${ }^{3}$ Reproductive Biotechnology Research Center, Avicenna Research Institute, ACECR, Tehran, Iran; ${ }^{4}$ Department of Molecular Medicine, Faculty of Advanced Technologies in Medicine, Iran University of Medical Sciences, Tehran, Iran; ${ }^{5}$ Department of Immunology, School of Public Health, Tehran University of Medical Sciences, Tehran, Iran; ${ }^{6}$ Reproductive Immunology Research Center, Avicenna Research Institute, ACECR, Tehran, Iran; ${ }^{7}$ Cellular and molecular research center, Iran University of Medical Sciences, Tehran, Iran

*For correspondence: mt.joghataei@yahoo.com; joghataei@iums.ac.ir

[Abstract] Rat transient middle cerebral artery occlusion (tMCAO) model is one of the most commonly used animal models in ischemic stroke studies. In the model, increasing safety and efficacy of therapeutic agent administration, such as stem cells and drugs directly to the ischemic brain using the internal carotid artery (ICA) is essential, because using the common carotid artery (CCA) for injection can close CCA completely and cause many complications after tMCAO surgery. Also, the pterygopalatine artery (PPA) is an arterial branch of the ICA that supplies blood circulation of the external part of the brain and removing the blood circulation of the PPA is required for more complete induction of ischemia to the brain. Herein, we present the insertion of intra-arterial catheter in the ICA via the external carotid artery (ECA) after the PPA in rats subjected to tMCAO surgery.

Keywords: Drug delivery, tMCAO model, Intra-arterial injection, Internal carotid artery, Ischemic stroke

[Background] Rat ischemic stroke models have been used substantially to evaluate the benefit of therapeutic agents and stem cells and to study the underlying mechanisms (Rousselet et al., 2012; Azedi et al., 2017). Since ischemic stroke in patients is mostly caused by the occlusion of the middle cerebral artery (MCA), intraluminal suture middle cerebral artery occlusion (MCAO) has become the most commonly used models in the investigation of stroke in rodents such as rat and mice (Longa et al., 1989; Wang-Fischer, 2008; Tajiri et al., 2013; Fluri et al., 2015).

Method of intra-arterial delivery in stroke treatment is used in various studies and shows high safety and efficacy (Cloft et al., 2009; Chua et al., 2011; Crumrine et al., 2011; Azedi et al., 2019). In fact, Stem cells or drugs can be injected into the internal carotid artery (ICA) via common carotid artery (CCA) for targeted delivery into the reperfused brain tissue (Li et al., 2010; Misra et al., 2012; Du et al., 2014). However, after cutting the CCA for any interventions or injections, it has to be closed completely; or many complications for the animal, like increasing the mortality rate, can occur. Also, because the pterygopalatine artery (PPA) is a branch of the ICA, blood or any drug solutions will shunt into the PPA 
supplied region during any injection to the ICA and the efficacy of treatment may be reduced. Therefore, most researchers occluded the PPA when they performed intra-arterial injection (Guo et al., 2013); however, because of deep location and poor access to this artery, ligation of the PPA is very hard and often causes complications (Chen et al., 2008). In this protocol, we describe a simple method for intra-arterial injection to the ICA via external carotid artery (ECA) to the ischemic brain without manipulating the PPA after IMCAO surgery. You can see a schematic view of this procedure in Figure 1.

A

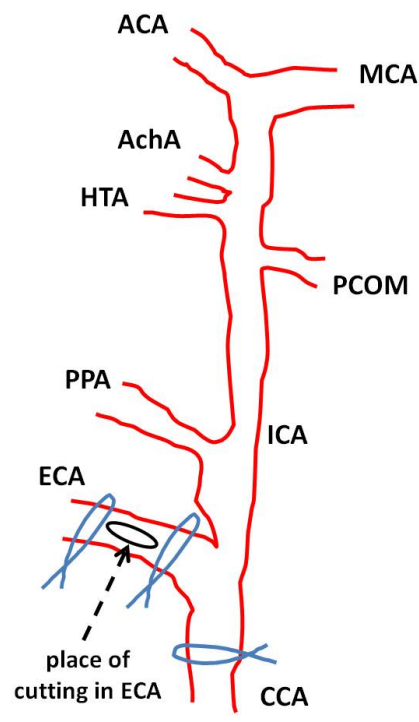

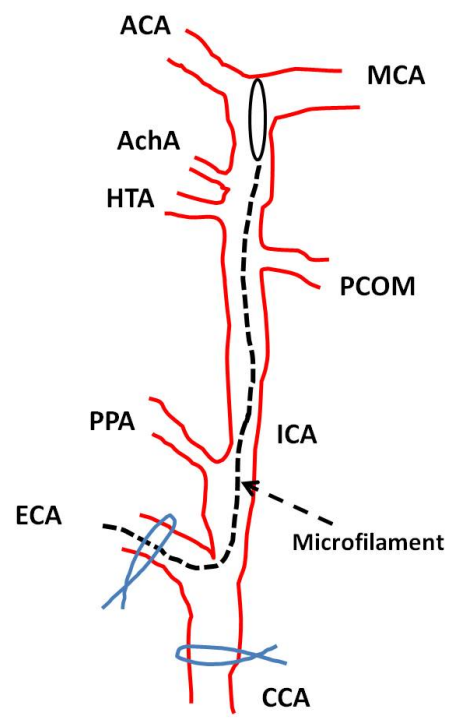

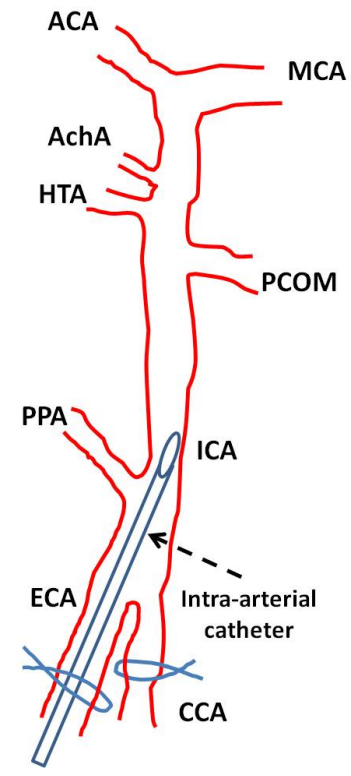

Figure 1. The schematic view of tMCAO surgery and insertion of intra-arterial catheter in ICA. A. The place of cutting in ECA. B. Insertion of microfilament for occluding MCA. C. Insertion of intra-arterial catheter after PPA branch. ACA: Anterior cerebral artery. AchA: Anterior choroid artery. CCA: Common carotid artery. HTA: Hypothalamic artery. ICA: Internal cerebral artery. MCA: Middle cerebral artery. PCOM: Posterior communication artery. PPA: Pterygopalatine artery.

\section{Materials and Reagents}

1. Cannula-24G $\times 19 \mathrm{~mm} \times 50$ (Terumo, catalog number: SR+DS2419PX)

2. Syringe (BD, catalog number: 328411)

3. Microfilament with round tip and silicon coating (Doccol, catalog number: 403734PK5Re)

4. 4-0 silk suture (Supa, catalog number: S64019B3)

5. 3-0 nylon suture (Supa, catalog number: N63026B3)

6. Sterile Gauze pad (Safa Teb, catalog number: 1307018)

7. Wistar rat (11-week-old)

8. Eye cream with vitamin A (Sina Darou, catalog number: 1228039225)

9. Ketamine (Rotexmedica, catalog number: 159-85-34830-00)

10. Xylazine (Interchemie, catalog number: Rp399) 
11. Lidocaine 10\% (Iran Darou, catalog number: 1228089381)

12. $0.25 \%$ Bupivacaine (AstraZeneca, catalog number: 0186-1027-01)

13. Ampicillin (GC Hanford, catalog number: 10515-946-25)

14. Alcohol (Merck, catalog number: 100983.2500)

15. Ketamine/xylazine solution (see Recipes)

\section{Equipment}

1. Balance (Sartorius, catalog number: LP6200S)

2. Stereo microscope (ZTX-E zoom, 10x-40x)

3. Rodent surgery board (VWR, model: 10718-012)

4. Rectal thermometer (Welch Allyn, model: SureTemp Plus 690)

5. Vessel clip (Roboz, catalog number: RS-5420)

6. Microdissecting spring scissor (Roboz, catalog number: RS-5600)

7. Toothless tweezers with straight (Roboz, catalog number: RS-4972)

8. Toothless tweezers with curve head (Roboz, catalog number: RS5079)

9. Scissors (Roboz, catalog number: RS6762)

10. Needle holder (Roboz, catalog number: RS-7860L)

11. Tooth head forceps (Roboz, catalog number: RS5112)

12. Microdissection retractor (Roboz, catalog number: RS6515)

13. Thermal blanket (ALA Scientific, catalog number: HEATINGPAD-1/2)

\section{Procedure}

A. Animal preparation

1. Examine the rats for gross abnormalities and behavioral deficits prior to anesthesia and operation.

2. Weigh each rat and then anesthetize in an appropriate dose of Ketamine $(80 \mathrm{mg} / \mathrm{kg}) / \mathrm{Xylazine}$ (7 mg/kg). Rats with 270-310 g are appropriate for surgery.

3. Put the rat in a supine position on a gauze pad lying over a thermal blanket on surgery board to maintain body temperature of $37 \pm 0.5^{\circ} \mathrm{C}$ during the surgery. Check the rat body temperature during the surgical procedures using a rectal thermometer.

4. Fix the forelimbs on the surgery board carefully.

B. Cervical dissection

1. Apply $70 \%$ alcohol on the anterior neck over the front part of the neck for sterilization and disinfection. 
2. Apply $10 \%$ lidocaine solution for local anesthesia of neck area. Besides, lidocaine avoids high activity of the vagus nerve and imbalance of sympathetic nerves that can occur during the separation of vagus nerve from carotid sheath, leading to decrease of animal survival.

3. Place eye cream on both eyes to avoid corneal drying.

4. Make a midline incision using scissors through the skin from the manubrium of the sternum to the area under the jaw.

5. Divide the right and left submandibular glands under microscope guidance and dissect the gland on the side of arterial surgery (right or left) to release it from the surrounding fascia. The carotid triangle should be visible at this step. Digastrics muscle is a landmark for finding carotid triangle (Figure 2).

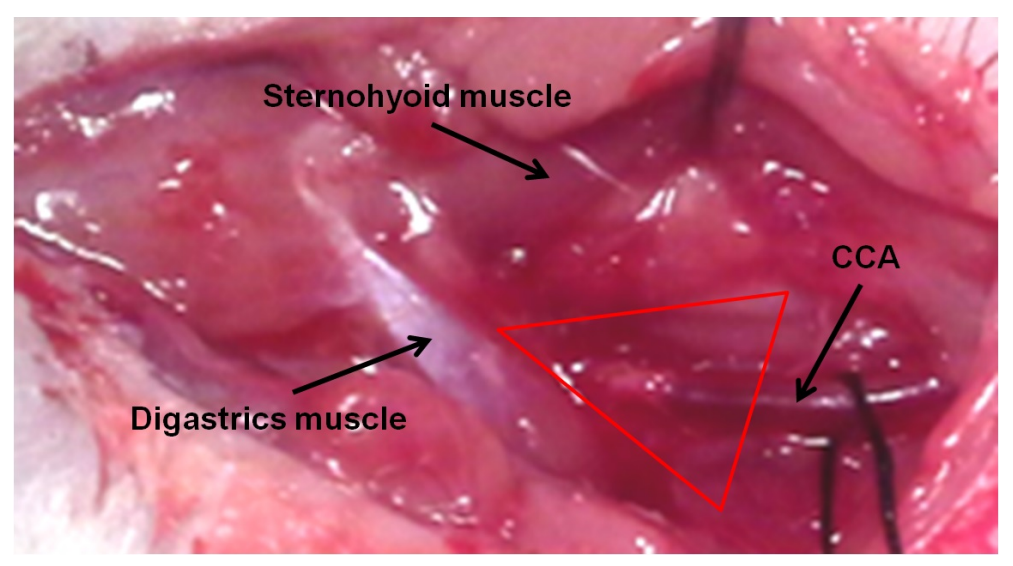

Figure 2. The carotid triangle and the location of common carotid artery

6. Divide the subjected part of the sternohyoid muscle to disrobe the underlying carotid sheath.

7. Dissect the carotid sheath, carefully isolate the common carotid artery and separate it from the vagus nerve and its branches, which are located lateral to it. Never damage the vagus nerve and its sheath. Just separate it from carotid artery (Figure 3). 


\section{b̆̈o-protocol}

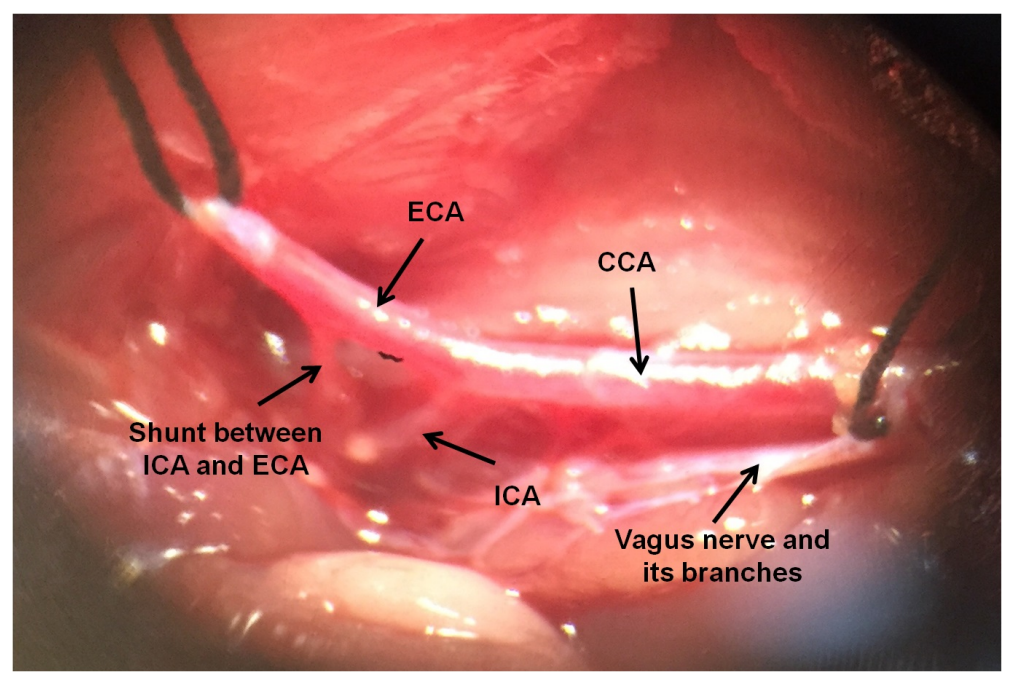

Figure 3. The location of common carotid artery, internal carotid artery and external carotid artery. Vagus nerve and its branches can be seen.

C. TMCAO method

1. Put a loose collar suture (4-0 silk) around the CCA.

2. Find the ECA and dissect it as close to the bifurcation as possible. It will help avoid bleeding from ECA branches.

3. Separate the bifurcation of the ICA and ECA. A permanent suture is located around the ECA, as distally as possible, and another temporary suture slightly tight is placed on the ECA distal to the bifurcation.

4. Temporarily close the CCA with a collar suture.

5. Temporarily close the ICA with a vessel clip to prevent retrograde flow when arterotomy is done (Figure 4).

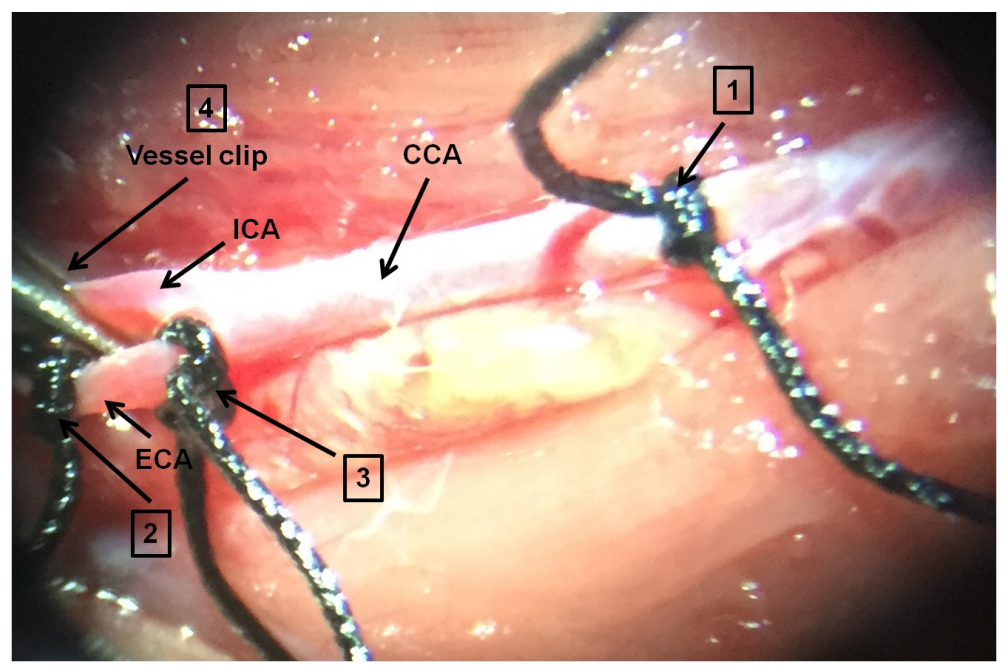

Figure 4. Making MCAO model. 1. Close CCA by vessel clip or temporary (loosely) suture; 2. Close ECA by a permanent (tightly) suture; 3 . Close ECA by temporary suture (slightly tight); 4 . Close ICA by vessel clip temporarily. 


\section{bĭ̈-protocol}

6. Cut a small hole (arterotomy) using microscissors into the ECA between permanent and temporary sutures (Figure 5).

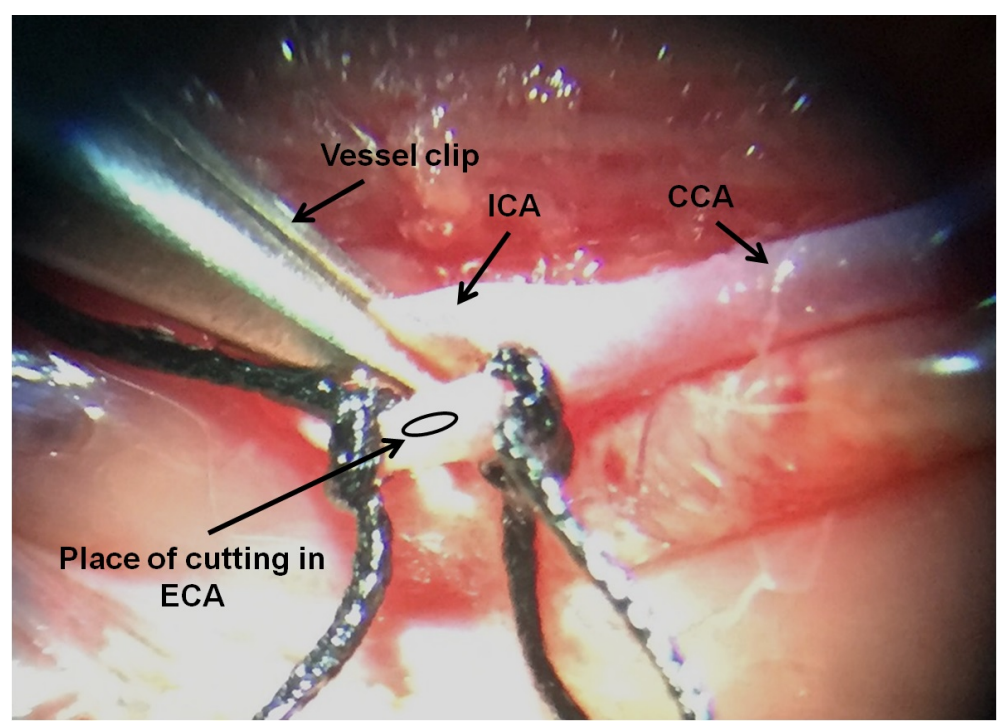

Figure 5. The place of cutting in ECA for inserting the microfilament. It is between two ECA sutures.

7. Introduce the microfilament into the ECA stump at the site of arteriotomy and advance the suture into the CCA.

8. Totally, cut the ECA distal to the permanent suture and turn the microfilament into the ICA. The suture is tightly tied around the monofilament to avoid bleeding (Figure 6).

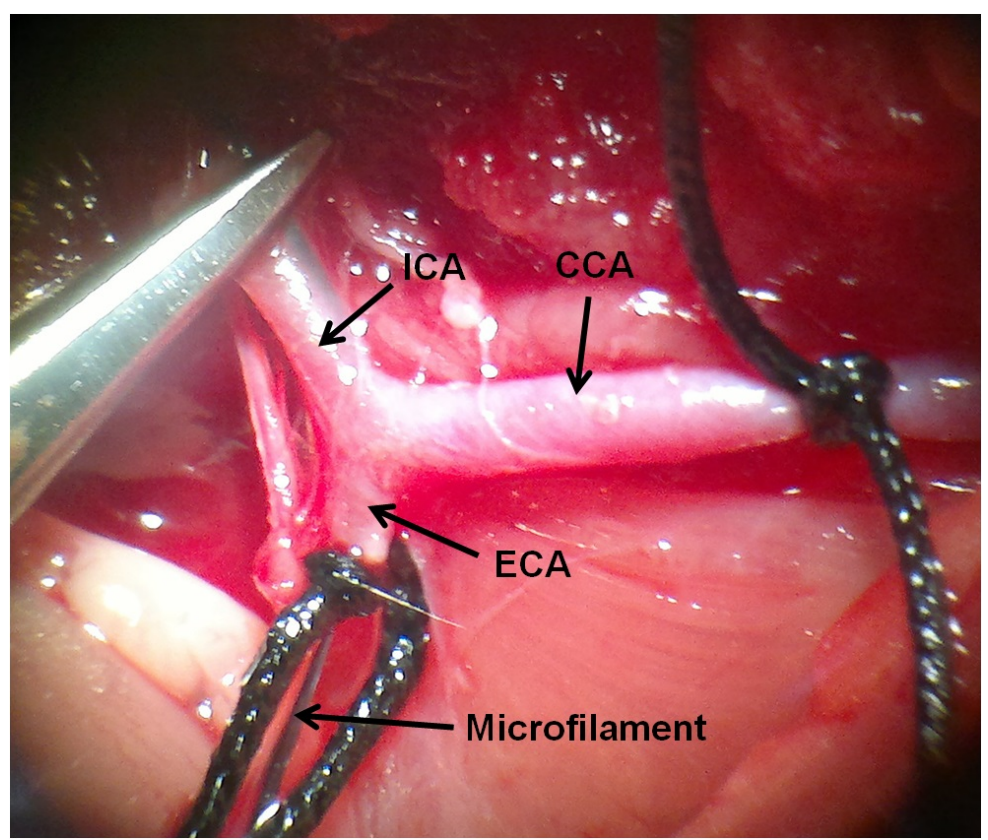

Figure 6. Inserting the microfilament into the ICA via the ECA 


\section{bĭo-protocol}

9. Gently advanced the microfilament from the ECA to the ICA to within about $18-20 \mathrm{~mm}$ from the bifurcation. At this point, the microfilament is blocking the origin of MCA. About a 5-7mm length of the microfilament remains outside, to be withdrawn to permit reperfusion. Keep away from inserting the microfilament into the PPA (Figure 7).

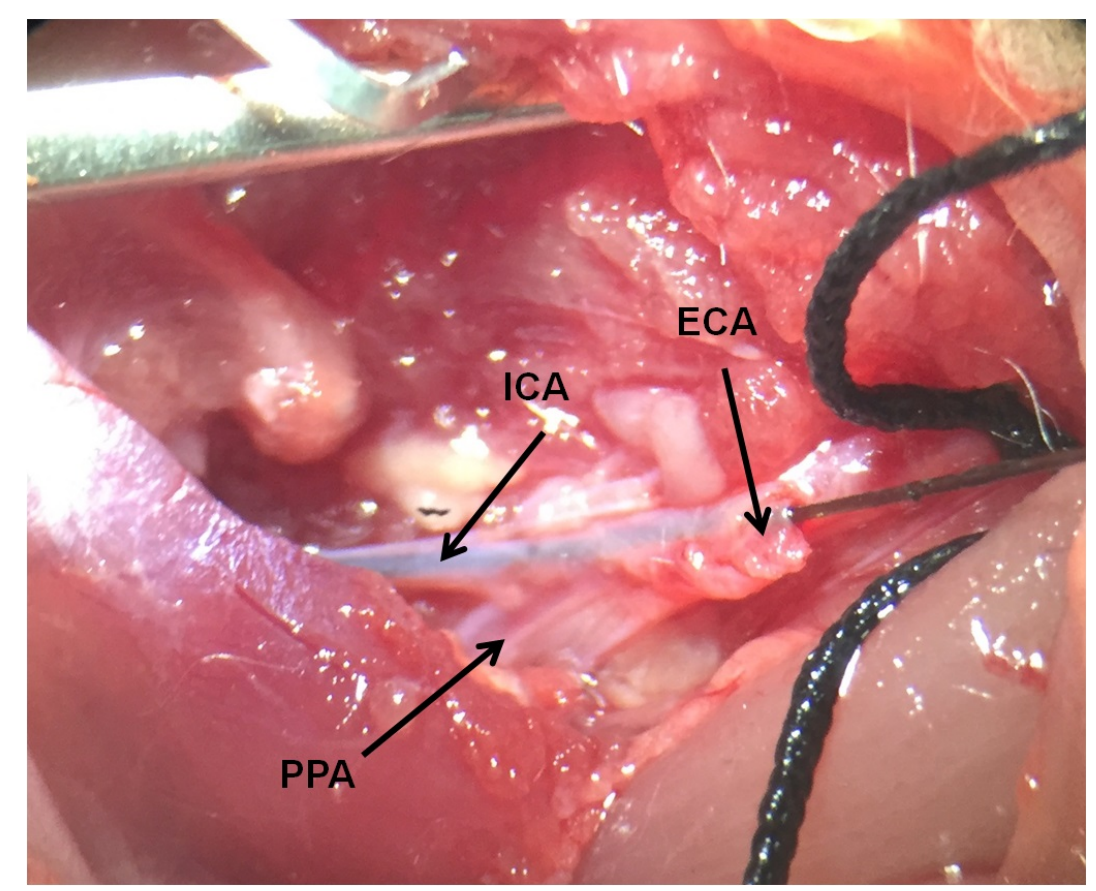

Figure 7. The place of PPA as a branch of ICA. The microfilament must be at the ICA through the ECA.

10. Remove the suture of CCA for starting ischemic phase (Figure 8).

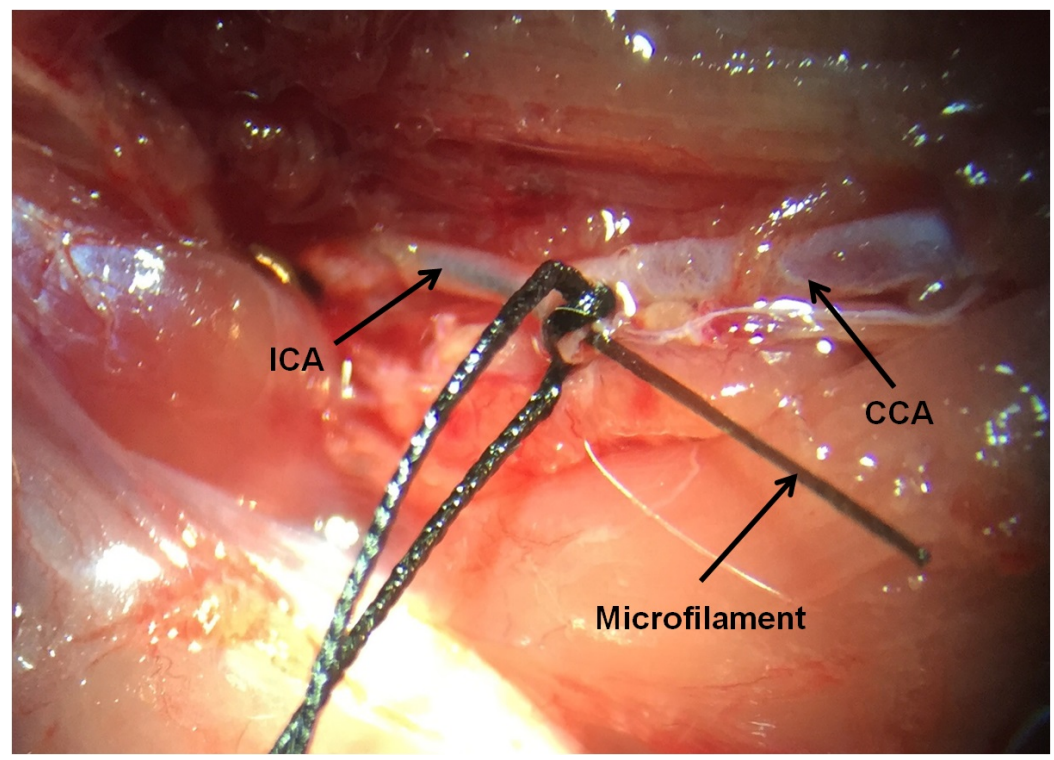

Figure 8. Some part of the microfilament remains outside to be withdrawn to allow reperfusion. The tip of microfilament blocks the origin of MCA. CCA is open. 
11. For starting reperfusion phase and removing microfilament, after ischemia (time of ischemia depends on your research) pull back the microfilament until resistance is felt, indicating that the tip is in the ECA stump.

12. Place a suture on the CCA to reduce blood flow, and then pull out the entire microfilament.

D. Inserting the catheter in the ICA

1. Insert the catheter into the ECA, then advanced into the ICA and further PPA (Figure 9). The catheter is $16.1 \mathrm{~mm}$ and inside of the ICA from the ECA stump is about $18-20 \mathrm{~mm}$. It is not needed to insert whole of the catheter. The PPA is about 4-6 mm after the ECA stump. So, advance the catheter to the ICA about 8-10 $\mathrm{mm}$ (depend on the animal size). Be careful about the variations in some arteries among rats. Some rats have a different place of ECA-ICA bifurcation. So, the size of the inside of the ICA from ECA stump can be different.
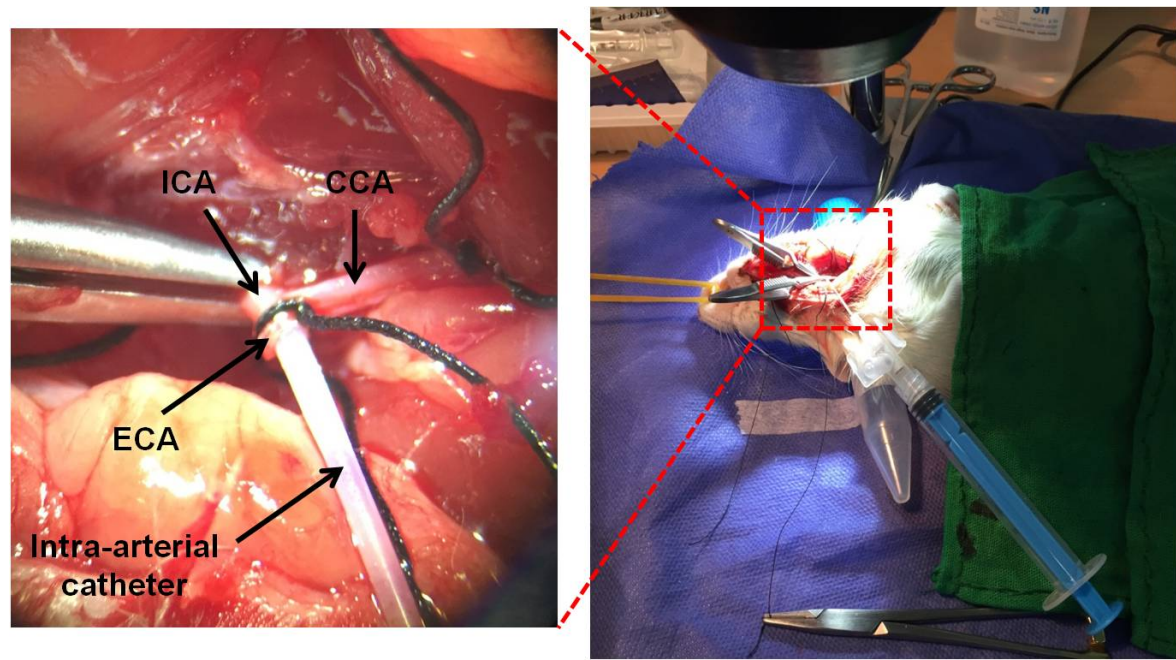

Figure 9. Inserting an intra-arterial catheter into the ICA after PPA. Injection can be performed with a syringe.

2. Place a collar suture around the ECA for preventing catheter removing.

3. Connect a syringe with the injected solution (maximum volume: $1 \mathrm{ml}$ )

4. Gently, inject the solution during $2 \mathrm{~min}$ for preventing brain damage.

5. Remove the catheter slowly.

6. The suture on the ECA is tightly tied.

7. Close the skin with a nylon suture (3-0) by using needle holder and forceps.

8. Give the rat locally $0.25 \%$ bupivacaine to reduce postoperative pain.

9. To prevent infection, use a single dose of $150 \mathrm{mg} / \mathrm{kg}$ Ampicillin (subcutaneously) just after surgery. Using for the long term is not recommended.

10. Put the rat in a cage with an appropriate temperature $\left(37^{\circ} \mathrm{C}\right)$ until the rat wakes up. The animal usually regains consciousness in 15-20 min. 


\section{Data analysis}

After inducing ischemia by MCA occlusion, it is possible to determine the infarct size by MRI Scanning and if it is appropriate, you can start to insert the catheter in the ICA for drug delivery. (Figure 10).

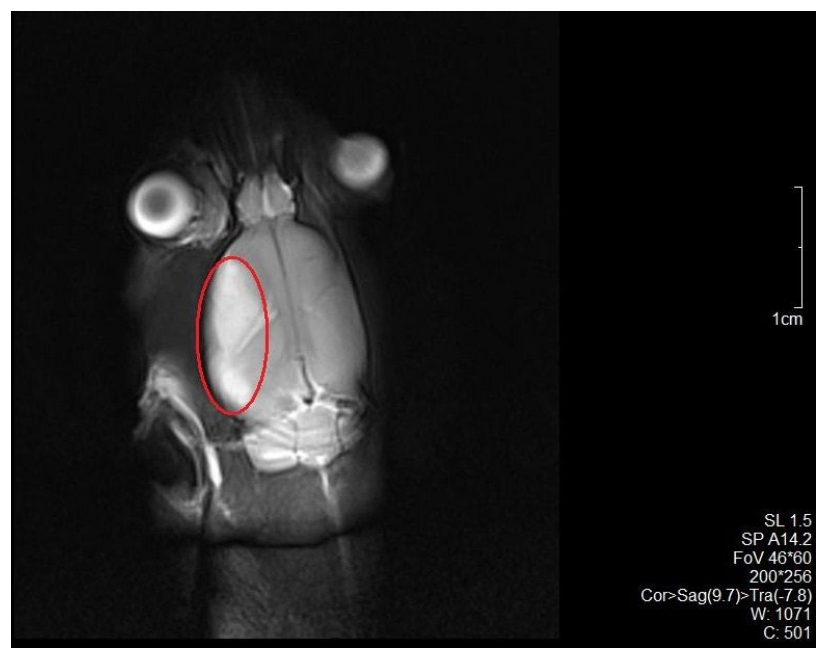

Figure 10. The MRI scans of a rat subjected to MCAO. The infarct area around the territory of MCA on the left side of rat brain can be seen. The red circle indicates the infarct area.

\section{Notes}

During the surgery, avoid manipulating vagus nerve because overactivation of this nerve leads to cardiac arrest. Furthermore, contraction of bronchioles can cause respiratory problems after the surgery and increase mortality. The size of the microfilament and weight of rat are very important. If you use small microfilament, ischemia can't occur, and if you use big microfilament, subarachnoid hemorrhage can happen.

\section{Recipes}

1. Ketamine/xylazine solution

Ketamine $(80 \mathrm{mg} / \mathrm{kg})$

Xylazine $(7 \mathrm{mg} / \mathrm{kg})$

Mix them and prepare Ketamine/Xylazine cocktail for surgical anesthesia

\section{Acknowledgments}

The authors are thankful to Nahid Aboutaleb, Physiology Research Center and Department of Physiology, Faculty of Medicine, Iran University of Medical Sciences, Tehran, Iran and Yasin Asadi, 
Laboratory of Learning and Memory, Physiology Research Center and Physiology Department, School of Medicine, Semnan University of Medical Sciences, Semnan, Iran for technical support, and Vice Chancellor of Research of Iran University of Medical Sciences, Tehran, Iran, for financial support. The authors as well thank the staff in the National Brain Mapping Lab, Tehran, Iran for allowing us to use their MRI scanner.

\section{Competing interests}

The authors declare that there was no conflict of interest or competing interests.

\section{Ethics}

All experiments were conducted according to the institutional guidelines with the protocol approved by the Committee for the Use of Live Animals in Teaching and Research, the Iran University of Medical Sciences and health ministry. The approval ID of the animal experiment in this protocol was 93-04-87-25297.

\section{References}

1. Azedi, F., Joghataei, M. T., Mousavizadeh, K. and Mehrpur, M. (2017). Novel methods for intra-arterial injection of stem cells to the ischemic brain: A neurosurgical approach. $J$ Cerebr Blood F Met 37(1_suppl):330-331.

2. Azedi, F., Mehrpour, M., Talebi, S., Zendedel, A., Kazemnejad, S., Mousavizadeh, K., Beyer, C., Zarnani, A. H., Joghataei, M. T. (2019). Melatonin regulates neuroinflammation ischemic stroke damage through interactions with microglia in reperfusion phase. Brain Research DOI: 10.1016/j.brainres.2019.146401.

3. Chen, Y., Ito, A., Takai, K. and Saito, N. (2008). Blocking pterygopalatine arterial blood flow decreases infarct volume variability in a mouse model of intraluminal suture middle cerebral artery occlusion. J Neurosci Methods 174(1): 18-24.

4. Chua, J. Y., Pendharkar, A. V., Wang, N., Choi, R., Andres, R. H., Gaeta, X., Zhang, J., Moseley, M. E. and Guzman, R. (2011). Intra-arterial injection of neural stem cells using a microneedle technique does not cause microembolic strokes. J Cereb Blood Flow Metab 31(5): 1263-1271.

5. Cloft, H. J., Rabinstein, A., Lanzino, G. and Kallmes, D. F. (2009). Intra-arterial stroke therapy: an assessment of demand and available work force. AJNR Am J Neuroradiol 30(3): 453-458.

6. Crumrine, R. C., Marder, V. J., Taylor, G. M., Lamanna, J. C., Tsipis, C. P., Scuderi, P., Petteway, S. R., Jr. and Arora, V. (2011). Intra-arterial administration of recombinant tissue-type plasminogen activator (rt-PA) causes more intracranial bleeding than does 
intravenous rt-PA in a transient rat middle cerebral artery occlusion model. Exp Transl Stroke Med 3(1): 10.

7. Du, S., Guan, J., Mao, G., Liu, Y., Ma, S., Bao, X., Gao, J., Feng, M., Li, G., Ma, W., Yang, Y., Zhao, R. C. and Wang, R. (2014). Intra-arterial delivery of human bone marrow mesenchymal stem cells is a safe and effective way to treat cerebral ischemia in rats. Cell Transplant 23 Suppl 1: S73-82.

8. Fluri, F., Schuhmann, M. K. and Kleinschnitz, C. (2015). Animal models of ischemic stroke and their application in clinical research. Drug Des Devel Ther 9: 3445-3454.

9. Guo, L., Ge, J., Wang, S., Zhou, Y., Wang, X. and Wu, Y. (2013). A novel method for efficient delivery of stem cells to the ischemic brain. Stem Cell Res Ther 4(5): 116.

10. Li, L., Jiang, Q., Ding, G., Zhang, L., Zhang, Z. G., Li, Q., Panda, S., Lu, M., Ewing, J. R. and Chopp, M. (2010). Effects of administration route on migration and distribution of neural progenitor cells transplanted into rats with focal cerebral ischemia, an MRI study. $J$ Cereb Blood Flow Metab 30(3): 653-662.

11. Longa, E. Z., Weinstein, P. R., Carlson, S. and Cummins, R. (1989). Reversible middle cerebral artery occlusion without craniectomy in rats. Stroke 20(1): 84-91.

12. Misra, V., Ritchie, M. M., Stone, L. L., Low, W. C. and Janardhan, V. (2012). Stem cell therapy in ischemic stroke: role of IV and intra-arterial therapy. Neurology 79(13 Suppl 1): S207-212.

13. Rousselet, E., Kriz, J. and Seidah, N., G. (2012). Mouse model of intraluminal MCAO: cerebral infarct evaluation by cresyl violet staining. $J$ Vis Exp (69).

14. Tajiri, N., Dailey, T., Metcalf, C., Mosley, Y. I., Lau, T., Staples, M., van Loveren, H., Kim, S. U., Yamashima, T., Yasuhara, T., Date, I., Kaneko, Y. and Borlongan, C. V. (2013). In vivo animal stroke models: a rationale for rodent and non-human primate models. Trans/ Stroke Res 4(3): 308-321.

15. Wang-Fischer, Y. (2008). Manual of stroke models in rats. CRC Press. 